\title{
Psicologia: Ciência e Profissão: 30 anos Registrando o Avanço da Psicologia Brasileira
}

\author{
Psicologia: Ciência e Profissão: 30 Years Registering the \\ Progress of the Brazilian Psychology \\ Psicología: Ciencia y Profesión - 30 años Registrando el \\ Progreso de la Psicología Brasileña
}

Maria Imaculada Cardoso Sampaio \& André Serradas

Universidade de São Paulo

Acácia Aparecida

Angeli dos Santos

Universidade São Francisco

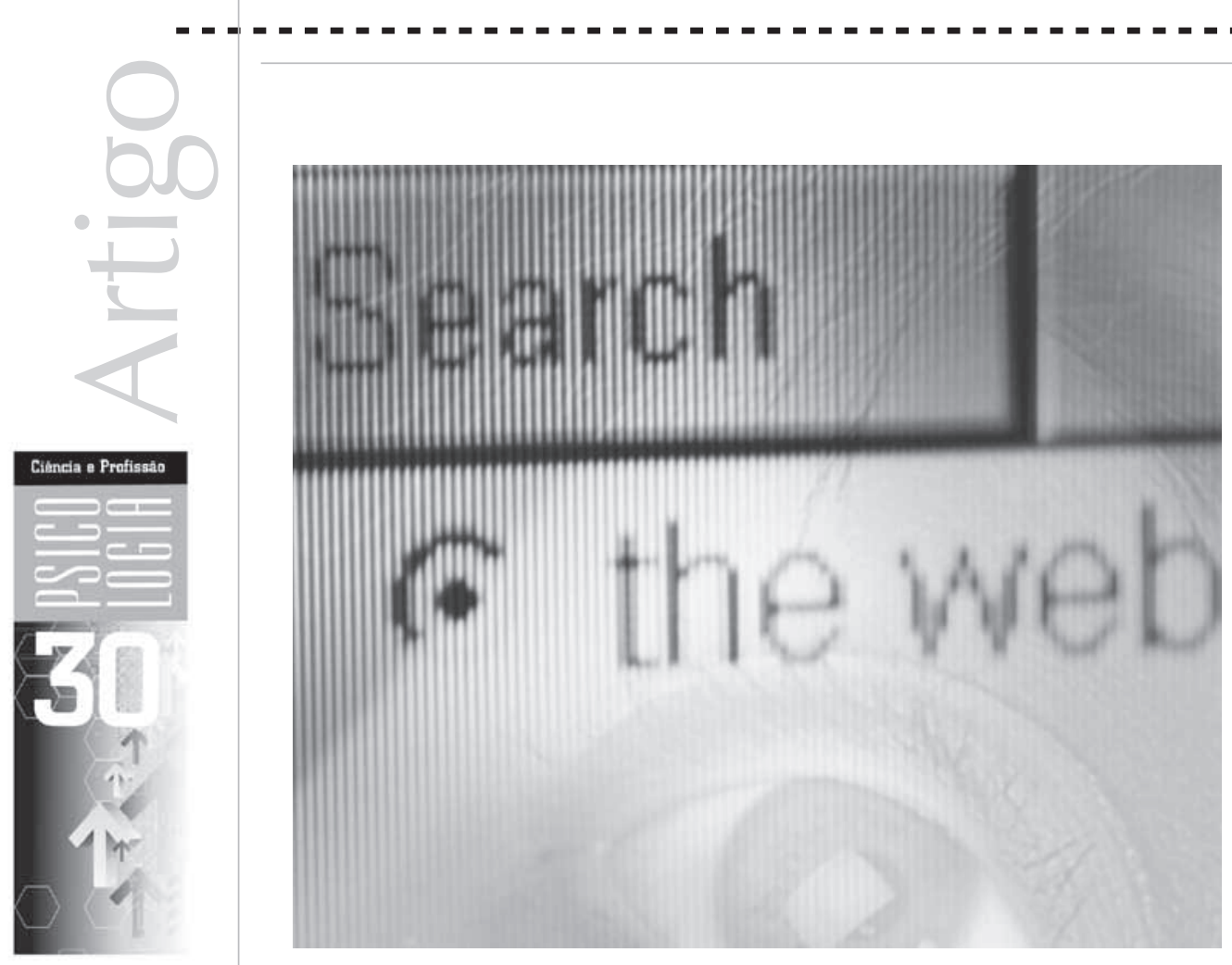


Resumo: Psicologia: Ciência e Profissão (PCP), publicada pelo Conselho Federal de Psicologia ao longo de 30 anos, registra o avanço da Psicologia, como ciência e profissão. O objetivo deste trabalho é relatar alguns aspectos que merecem destaque, a partir de 2005, quando PCP se consolida como uma revista científica, ganha a versão digital e se insere no movimento do acesso aberto. É feita também uma breve análise dos indicadores de citações da revista. As análises foram efetuadas a partir da leitura dos editoriais dos fascículos publicados do ano 2005 até o momento. Com base nos módulos de bibliometria do PePSIC e SciELO, além dos dados das referências da revista, foram levantados indicadores de citações recebidas e fornecidas por PCP. Os dados permitem afirmar que PCP vem aumentando o número de citações recebidas. A rede de revistas que mais citam PCP está publicada no PePSIC, demonstrando que publicações eletrônicas ganham maior visibilidade na comunidade científica. Ao completar 30 anos, a proposta da revista é o comprometimento com a Psicologia voltada para aliviar o sofrimento humano, mas também dedicada ao avanço da ciência psicológica. O conhecimento documentado em revistas científicas e o impacto que tais publicações representam para o saber universal é algo que deve ser abordado, discutido e entendido, assim, estudos dessa natureza devem ser incentivados.

Palavras-chave: Revistas científicas. Psicologia. Conhecimento científico. Indicadores de produção científica.

\begin{abstract}
Psychology: Science and Profession (PCP) published by the Psychology Federal Council throughout the last 30 years has been tracking the progress in the field both in terms of science and profession. The goal of this study is to report several relevant aspects starting from 2005 when PCP established itself as a scientific journal while it became available in an electronic version joining the open access movement. A brief analysis of the references and indicators is done at the same time. This analysis has taken place based on the editorial contents of the volumes published since 2005. Based on the PePSIC and SciELO bibliometric modules' references given and received by the PCP have been documented on top of the journal references. The most active journal network in terms of references to the PCP is published in the PePSIC, illustrating that electronic journals are subjected to higher visibility in the scientific community. At the 30th anniversary the journal's commitment with Psychology is oriented to the relief of human suffering but also dedicated to the advancement of the psychological science. The knowledge captured in scientific journals and the respective impact that such contents represent to the universal knowledge should be approached, discussed and understood; therefore research activities of this nature should be fostered.
\end{abstract}

Keywords: Scientific journals. Psychology. Scientific knowledge. Scientific publication Indicators.

Resumen: Psicologia: Ciência e Profissão (PCP) es una publicación del Conselho Federal de Psicologia y desde hace 30 años registra el avance de la Psicología, en cuanto ciencia y profesión. El objetivo de este trabajo es relatar algunos aspectos que merecen atención a partir del 2005 cuando PCP se consolida como una revista científica, empieza a tener una versión digital y pasa a formar parte del movimiento del acceso abierto. Además se hace un breve análisis de los indicadores de citas de la Revista. Los análisis se llevaron a cabo mediante la lectura de los editoriales de los fascículos que se han publicado del 2005 hasta la fecha. Basándose en los módulos de bibliometría del PePSIC y SciELO, además de los datos de las referencias de la Revista, se obtuvo también el levantamiento de los indicadores de citas recibidas y cedidas por PCP. El análisis de los datos permite afirmar que PCP ha aumentado el número de citas recibidas. La red de revista que más la cita está publicada en el PePSIC, lo que señala que las publicaciones electrónicas tienen una mayor visibilidad en la comunidad científica. Al cumplir los 30 años, el objetivo de la Revista es el compromiso con la Psicología que busca aliviar el sufrimiento humano y también dedicada al avance de la ciencia psicológica. El conocimiento documentado en revistas científicas y el impacto que tales publicaciones representan para el saber universal es algo que se debe abordar, discutir y entender, por lo tanto, estudios de esa naturaleza deben de ser incentivados.

Palabras clave: Revistas científicas. Psicología. Conocimiento científico. Indicadores de producción científica.

Publicada desde 1979 pelo Conselho Federal de Psicologia, a revista Psicologia: Ciência e Profissão (PCP) vem registrando a evolução da área e suas principais transformações. Ao comemorar 30 anos, a publicação atingiu maturidade e reconhecimento entre os psicólogos, estando inserida definitivamente no fazer da Psicologia como ciência e profissão no Brasil.

O objetivo deste trabalho é relatar alguns aspectos que merecem destaque, a partir de 2005, quando PCP se consolida como uma 
O editorial do

número 1 do

volume 25

comemora

a entrada da

revista no portal

de Periódicos

Eletrônicos de

Psicologia (PePSIC) revista científica, ganha a versão digital e se insere definitivamente no movimento do acesso aberto. É feita também uma breve análise dos indicadores de citações da revista no período. Essa nota vai ao encontro, em alguns aspectos, ao artigo de Campos e Bernardes (2005), que fizeram um registro, com muita propriedade, da história da revista, desde seu primeiro fascículo, no ano 1979, até o ano 2004, quando completou 25 anos de publicação.

O volume 25 de PCP apresenta mudanças no projeto gráfico. A revista deixou de publicar imagens no meio do texto, abrindo espaço para que os autores ilustrassem seus artigos com tabelas e gráficos, características do texto científico. Embora distanciando-se do modelo jornalístico, PCP manteve um laço com a suavidade dos fascículos anteriores, publicando imagens ilustrativas na página inicial dos artigos. Mantendo uniformidade na composição do conselho editorial, a publicação mantém a preocupação com a representatividade regional, que faz com que a comissão editorial inclua psicólogos das cinco Regiões do País. Nesse volume, a nominata dos pareceristas ad hoc passa a ser publicada uma vez por ano, em contraposição aos volumes anteriores, que traziam a lista de consultores em todos os fascículos.

O editorial do número 1 do volume 25 comemora a entrada da revista no portal de Periódicos Eletrônicos de Psicologia (PePSIC). A fonte de informação, recém-lançada na Biblioteca Virtual em Saúde - Psicologia (BVS-Psi ULAPSI Brasil), surgia como uma excelente alternativa para popularizar o acesso às revistas de Psicologia. Nas palavras da editora, "é a total democratização da informação, e a Psicologia sai à frente, mais uma vez".

No segundo fascículo de 2005, o editorial destaca o I Congresso Latino-Americano de Psicologia, promovido pela União Latino- americana de Entidades de Psicologia (ULAPSI), que iniciava uma ação em prol da integração da Psicologia latino-americana, "seja por meio de pesquisas que ampliem o conhecimento sobre os nossos problemas, seja pela convergência de atuação de profissionais que compartilhem das mesmas preocupações." Nesse contexto, a organização e a disseminação do conhecimento através da Biblioteca Virtual em Saúde - Psicologia da ULAPSI (BVS-Psi ULAPSI) eram apontadas como um importante caminho a ser percorrido.

Os textos agraciados com o prêmio Pedro Parafita Bessa-Subjetividade, Encarceramento e Sistema Prisional: Desafios para a Psicologia, instituído pelo Conselho Federal de Psicologia, compõem o número 4 do volume 26. De acordo com o editorial daquele fascículo, o tema do prêmio, raramente debatido nos cursos de Psicologia, recebeu manifestação de interesse pela comunidade, "revelando que o desafio do debate sobre o sistema prisional se mostrou atrativo e instigador, podendo-se afirmar que ele já configura um dos campos de trabalho da Psicologia".

O aumento do número de profissionais registrados no Sistema Conselhos e a ampliação do número de artigos publicados por PCP impossibilitaram o envio da revista impressa ao conjunto de psicólogos do País. Assim, no ano 2007, o editorial do volume 27, fascículo 1, explica a restrição da distribuição a cinco mil exemplares que seriam encaminhados às bibliotecas de todos os cursos de Psicologia e às instituiçõesreferência na área. A revista explica que "essa medida, em um primeiro momento, provocou certa retração no número de artigos submetidos à publicação, visto que muitos autores entenderam que a revista não teria mais o potencial de divulgação que possuía até então". Estratégias como a ampliação do número de exemplares comemorativos aos 25 anos para 25 mil, a ampla divulgação da 
revista em congressos de Psicologia, além da recuperação dos artigos completos no PePSIC, fizeram com que PCP recuperasse seu fôlego e "o envio de artigos saltou de 64 trabalhos, em 2004, para 84, em 2005, e para 110, em 2006; no ano 2007, foram submetidos 126 trabalhos; em 2008, 167 textos foram recebidos.

No primeiro fascículo de 2008, PCP comemora a nova aparência da capa e do miolo da revista, que agora utiliza papel reciclado, refletindo a afinidade da publicação "com a busca pelo desenvolvimento sustentável que inclui principalmente a preservação da natureza", explica o editorial do fascículo 1 , volume 28. Nesse ano, a revista também passa a utilizar as normas da American Psychological Association (APA) e inclui colegas latino-americanos no conselho consultivo, em um esforço para estreitar os laços de compromisso com a Psicologia de países que têm realidades sociais muito semelhantes. O balanço da gestão editorial do ano 2008, publicado no editorial do fascículo 4, volume 28 , dá conta que, dos 167 textos submetidos no ano, $24 \%$ são de assuntos ligados à Psicologia social. "Outros $21 \%$ referem-se a temas de saúde mental. Em terceiro lugar, com $18 \%$, estão os trabalhos relacionados à Psicologia clínica e hospitalar. Um percentual grande de textos, que soma $15 \%$, foi dedicado à Psicologia organizacional e do trabalho. Outras áreas aparecem, havendo textos relacionados à Psicologia escolar e educacional (7\%), à psicanálise (5\%), à avaliação psicológica (2\%) e à Psicologia jurídica (1\%). Os dados demonstram que PCP é vista como um órgão de disseminação da multidisciplinaridade da área. É no volume 28 que a revista passa a publicar artigos advindos da América Latina, facilitando a interlocução ente os autores e os pesquisadores da região, que têm poucos órgãos publicadores de seus países indexados em bases de dados internacionais. A iniciativa busca também alterar o panorama da carência de citações cruzadas entre os autores da região, que poderá ser observado logo à frente na análise das citações de PCP nos indicadores bibliométricos do PePSIC.

A adoção do Sistema PePSIC de publicação é registrada no editorial do número 1 , volume 29. “A Psicologia: Ciência e Profissão está entre as primeiras revistas da área da Psicologia a adotar essa metodologia, com o objetivo de tornar o procedimento de editoração, além de mais rápido, também mais transparente, pois o autor poderá acompanhar o seu trabalho, identificando em que fase do processo editorial se encontra". No ano 2009, os resumos passam a ser publicados em espanhol, além do português e do inglês, ampliando o acesso aos artigos de psicólogos e pesquisadores dos demais países da América Latina. No ano 2009, PCP teve toda a coleção digitalizada, marcada e publicada no PePSIC, ampliando o acesso a todos os artigos publicados desde 1979.

\section{Indicadores bibliométricos de PCP, de acordo com o PePSIC, no período 2005-2009}

Dentre os indicadores bibliométricos mais utilizados, e que causam muita polêmica entre a comunidade científica, estão os índices de impacto e citação. Até poucos anos atrás, as fontes de informação utilizadas para essas análises eram as bases de dados Science Citation Index (SCl) e o Social Science Citation Index (SCCI), desenvolvidas pelo Institute for Scientific Information (ISI) e atualmente mantidas pela Thomson Reuters (Barreto, 2006). Eugene Garfield, diretor emérito do ISI, criou o fator de impacto no ano 1955 e deu origem aos indicadores que até hoje sustentam grande parte dos requisitos que avaliam a produtividade dos cientistas. Dois fatores são referenciados na literatura sobre o tema: impacto dos trabalhos, indicador que mede o número de citações aos artigos 
publicados em revistas indexadas nas bases de dados da Web of Science (WOS), e impacto das revistas, que avalia o número de citações às revistas indexadas nas mesmas bases de dados. De acordo com Silva e Bianchi (2001), "O fator de impacto de uma revista num dado ano é o quociente entre o número de citações recebidas nesse ano pelos artigos publicados nos dois anos anteriores e o número de artigos publicados na revista naqueles dois anos" (p. 8). A base de dados que analisa o fator de impacto é o Journal Citacion Reports (JCR), que faz parte do conjunto de fontes de informação da WOS. Para que uma revista tenha presença no ranking do JCR, é necessário que seus artigos tenham recebido citações por outros autores nas revistas indexadas na WOS, o que não é fácil para as revistas dos países em desenvolvimento.

Até pouco tempo atrás, para que uma revista científica fosse valorizada, bastava estar indexada em uma boa base de dados. Atualmente, além de estar indexada, a revista precisa ser citada por outras revistas. As revistas de uma área formam uma rede de comunicação, e ter seus artigos referendados pela comunidade representa o reconhecimento do valor dos trabalhos ali publicados. No texto científico, a literatura é a base do argumento (premissas). A literatura é citada para que o leitor possa ter acesso aos textos originais que fundamentaram a discussão. Ao citar, o autor reconhece a obra do citado como contribuição que embasa o novo saber explicitado e oferece o registro das obras originais, guiando o leitor ao conhecimento anterior. Ao publicar um texto, desejamos que muitas pessoas consultem nossa obra e aprendam com ela para que a ciência avance. A citação é a prova que nosso texto cumpriu seu objetivo. "O fator de impacto reflete o uso que a comunidade científica está fazendo de certa revista" (Volpato, 2003, p. 58). Mesmo que seja para criticar (o que não é comum), a citação prova que o trabalho foi importante naquela discussão.

Nos últimos anos, surgiram outras fontes de informação capazes de analisar o número de citações de uma rede de revistas. É o caso da Scientific Electronic Library Online (SciELO), desenvolvida pela Fundação de Amparo à Pesquisa de São Paulo -FAPESP, em parceria com o Centro Latino-Americano e do Caribe de Informação em Ciências da Saúde - Bireme -, que abrange uma coleção selecionada de periódicos científicos brasileiros. O SciELO utilizou a mesma metodologia do ISI para criar o módulo de bibliometria, que gera relatórios de uso e citações, ampliando as possibilidades de avaliação do impacto das revistas brasileiras que não faziam parte da WOS.

Em 2004, a Psicologia, preocupada com a visibilidade das revistas brasileiras da área, adota o modelo SciELO de publicação e, em parceria com a BIREME e o Fórum Nacional das Entidades da Psicologia Brasileira (FENPB), sob a coordenação da Biblioteca Virtual em Saúde - Psicologia (BVS-Psi ULAPSI Brasil), cria o Portal de Periódicos Eletrônicos de Psicologia (PePSIC). No ano 2006, já inserido no contexto da produção científica do Brasil, o PePSIC é expandido para os demais países da América Latina e passa a publicar as revistas de diversos países da região. Com o fortalecimento do portal, a área ganha um novo elemento capaz de promover a análise das citações cruzadas entre as revistas de Psicologia. O PePSIC utiliza a mesma metodologia do SciELO para medir as citações de sua rede de revistas.

A PCP foi uma das primeiras revistas a fazer parte dessa rede de publicações. Como um exercício sobre o impacto da revista na comunidade, apresentamos, a seguir, uma breve discussão dos seus indicadores de citações de PCP, de acordo com o módulo de bibliometria do PePSIC. 


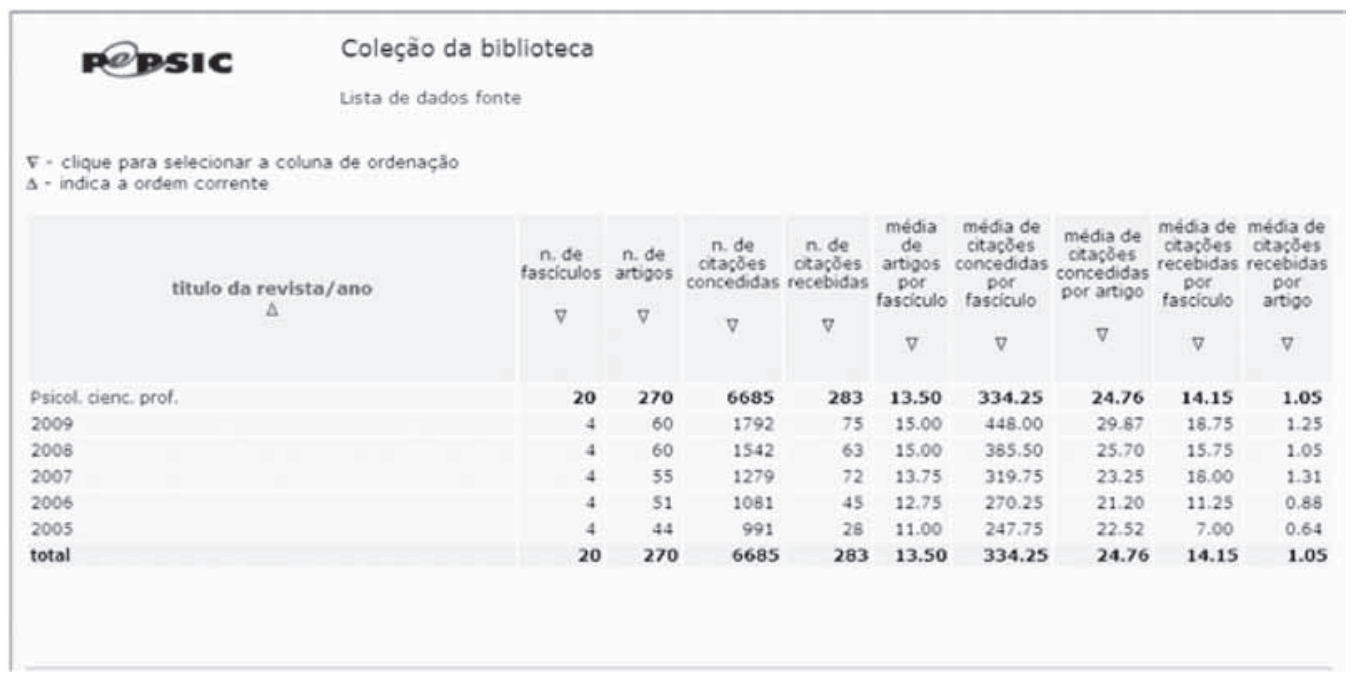

Figura 1. Dados fontes de PCP, obtidos no módulo de bibliometria do Portal de Periódicos Eletrônicos em Psicologia - PePSIC.

A periodicidade de PCP se manteve constante, com quatro fascículos nos anos de 2005 a 2009. O número de artigos aumentou consideravelmente, passando de 44 artigos publicados em 2005 para 60 no ano 2009. Em consequência, o número de citações concedidas e recebidas aumentou significativamente. Os dados comprovam o fortalecimento e a consolidação da publicação como fonte de informação da área.

O ranking a seguir registra os 50 títulos mais citados e o número de citações concedidas nas referências dos artigos publicados na revista PCP, no período de 2005 a 2009. Foram reunidas, pelo número de citações, os títulos que receberam até seis referências no período analisado.

Tabela 1. Ranking dos 50 títulos mais citados por PCP, no período de 2005 a 2009.

\begin{tabular}{lll}
\hline $\begin{array}{l}\text { Posição } \\
\text { no ranking }\end{array}$ & título & citações \\
\hline 1 & PSICOLOGIA: CIÊNCIA E PROFISSÃO & 69 \\
2 & PSICOLOGIA: REFLEXÃO E CRÍTICA & 64 \\
3 & ESTUDOS DE PSICOLOGIA (CAMPINAS) & 52 \\
4 & PSICOLOGIA EM ESTUDO & 47 \\
5 & CADERNOS DE SAÚDE PÚBLICA & 33 \\
6 & CIÊNCIA \& SAÚDE COLETIVA & 30 \\
6 & JOURNAL OF AUTISM AND DEVELOPMENTAL DISORDERS & 30 \\
6 & PSICOLOGIA: TEORIA E PESQUISA & 30 \\
7 & JOURNAL OF CONSULTING AND CLINICAL PSYCHOLOGY & 23 \\
7 & REVISTA DE SAÚDE PÚBLICA & 23 \\
8 & JOURNAL OF PERSONALITY AND SOCIAL PSYCHOLOGY & 20 \\
9 & AMERICAN PSYCHOLOGIST & 19 \\
9 & PERSONALITY AND INDIVIDUAL DIFFERENCES & 19 \\
9 & REVISTA BRASILEIRA DE PSIQUIATRIA & 19
\end{tabular}


continuação tabela 1

\begin{tabular}{|c|c|c|}
\hline $\begin{array}{l}\text { Posição } \\
\text { no ranking }\end{array}$ & título & citações \\
\hline 10 & FOLHA DE S. PAULO & 17 \\
\hline 10 & PSICOLOGIA \& SOCIEDADE & 17 \\
\hline 11 & JOURNAL OF VOCATIONAL BEHAVIOR & 15 \\
\hline 12 & PSYCHOLOGICAL BULLETIN & 14 \\
\hline 13 & REVISTA LATINO-AMERICANA DE ENFERMAGEM & 13 \\
\hline 13 & TEMAS EM PSICOLOGIA & 13 \\
\hline 14 & PEDIATRICS & 12 \\
\hline 14 & PSICO (PORTO ALEGRE) & 12 \\
\hline 15 & JOURNAL OF CLINICAL PSYCHOLOGY & 11 \\
\hline 15 & PSICO-USF & 11 \\
\hline 16 & JORNAL BRASILEIRO DE PSIQUIATRIA & 10 \\
\hline 16 & JOURNAL OF COUNSELING PSYCHOLOGY & 10 \\
\hline 16 & PSICOLOGIA USP & 10 \\
\hline 16 & PSICOLOGIA: TEORIA E PRÁTICA & 10 \\
\hline 16 & REVISTA BRASILEIRA DE ORIENTAÇÃO PROFISSIONAL & 10 \\
\hline 17 & CHILD DEVELOPMENT & 9 \\
\hline 17 & NEUROBIOLOGIA & 9 \\
\hline 17 & O ESTADO DE S. PAULO & 9 \\
\hline 17 & REVISTA DE PSIQUIATRIA CLÍNICA & 9 \\
\hline 18 & DEVELOPMENTAL PSYCHOLOGY & 8 \\
\hline 19 & ARCHIVES OF GENERAL PSYCHIATRY & 7 \\
\hline 19 & ARQUIVOS BRASILEIROS DE PSICOLOGIA & 7 \\
\hline 19 & BRITISH JOURNAL OF PSYCHIATRY & 7 \\
\hline \multirow[t]{2}{*}{19} & CAHIERS DE PSYCHOLOGIE COGNITIVE-CURRENT & \\
\hline & PSYCHOLOGY OF COGNITION & 7 \\
\hline 19 & CHILD ABUSE \& NEGLECT & 7 \\
\hline 19 & HEALTH PSYCHOLOGY & 7 \\
\hline 19 & JOURNAL OF CHILD PSYCHOLOGY AND PSYCHIATRY & 7 \\
\hline 19 & PSICOLOGIA ESCOLAR E EDUCACIONAL & 7 \\
\hline 20 & ANNUAL REVIEW OF PSYCHOLOGY & 6 \\
\hline 20 & BEHAVIOR ANALYST & 6 \\
\hline 20 & BULLETIN DE PSYCHOLOGIE & 6 \\
\hline 20 & CADERNOS DE PESQUISA & 6 \\
\hline 20 & JAMA-JOURNAL OF THE AMERICAN MEDICAL ASSOCIATION & 6 \\
\hline 20 & JOURNAL OF APPLIED BEHAVIOR ANALYSIS & 6 \\
\hline 20 & PSICOLOGIA ARGUMENTO & 6 \\
\hline 20 & PSYCHOSOMATIC MEDICINE & 6 \\
\hline 20 & JOURNAL OF GERONTOLOGY & 6 \\
\hline 20 & PAIDEIA & 6 \\
\hline 20 & ECONOMIA DE COMUNHÃO: UMA NOVA CULTURA & 6 \\
\hline 20 & BRAIN INJURY & 6 \\
\hline 20 & REVISTA DE APS & 6 \\
\hline 20 & REVISTA BRASILEIRA DE EDUCAÇÃO ESPECIAL & 6 \\
\hline 20 & AMERICAN JOURNAL OF PUBLIC HEALTH & 6 \\
\hline
\end{tabular}


Nos seis primeiros lugares do ranking das revistas mais citadas por PCP, estão revistas brasileiras reconhecidas pela comunidade. Na sétima posição, aparece um título altamente especializado. Ao analisar tais citações, observamos que apenas dois artigos, publicados no fascículo 1 do volume 29, concentram as 30 citações à revista Journal of Autism and Developmental Disorders. A observação nos leva a alertar que estudos de citações devem ser vistos com muita cautela, pois determinados artigos podem influenciar o número de citações e dar uma visão distorcida do impacto da publicação. Destacamos as 17 citações no jornal Folha de São Paulo e as 9 no Estado de São Paulo. Não temos nenhuma revista de Psicologia publicada em outro país da América Latina nas 20 posições do ranking.

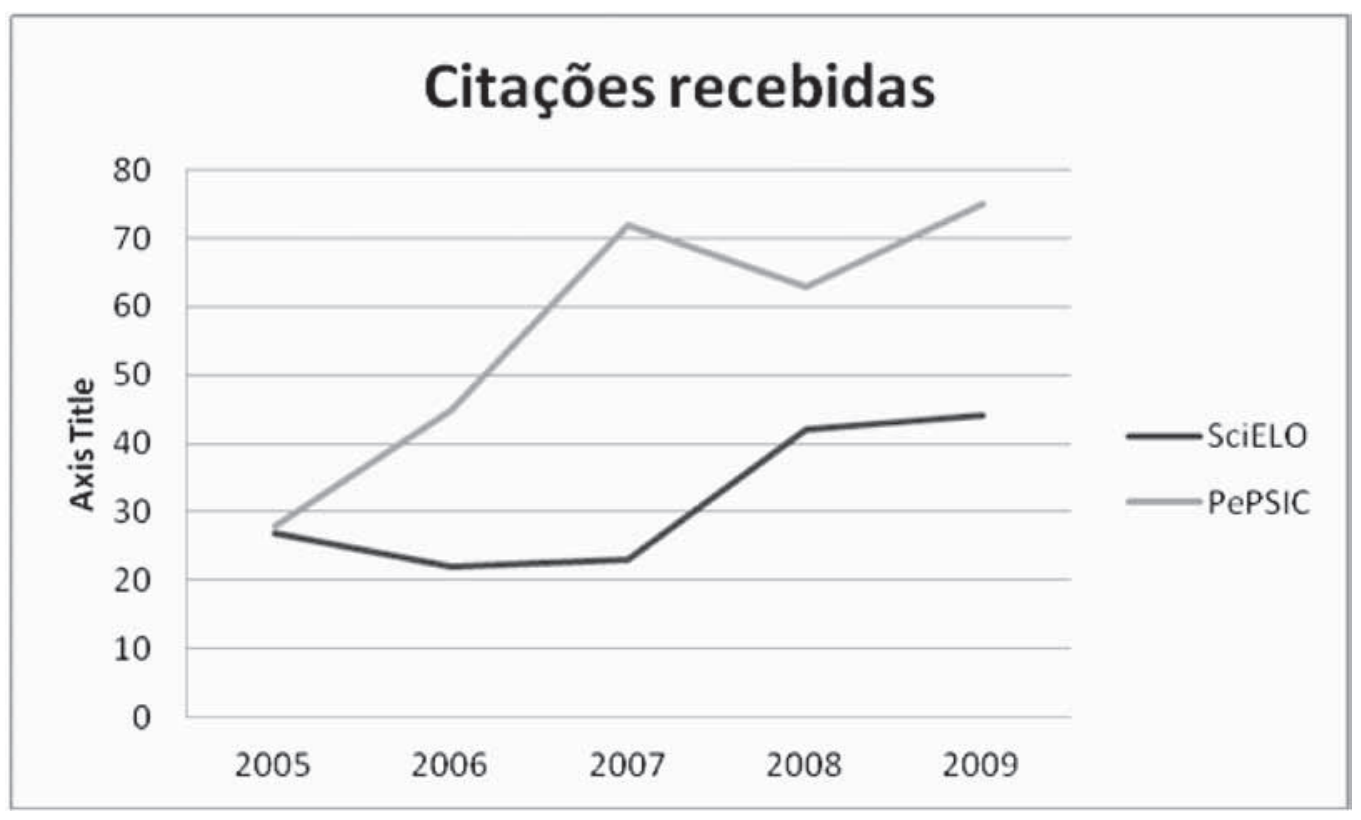

Figura 2. Citações recebidas pela revista PCP nos portais SciELO e PePSIC (2005-2009).

Tanto no SciELO quanto no PePSIC, é notório o aumento das citações recebidas por PCP. Notamos um avanço importante entre 2006 e 2008, que diminuiu em 2009. No PePSIC, as revistas são especializadas em Psicologia, enquanto no SciELO estão revistas de diversas áreas do conhecimento. Vale lembrar que esses dados são mutáveis, pois, a cada revista incluída no SciELO, ou PePSIC, que trouxer uma citação à PCP, o dados sofrerão alteração, e esse gráfico não será mais verdadeiro. 


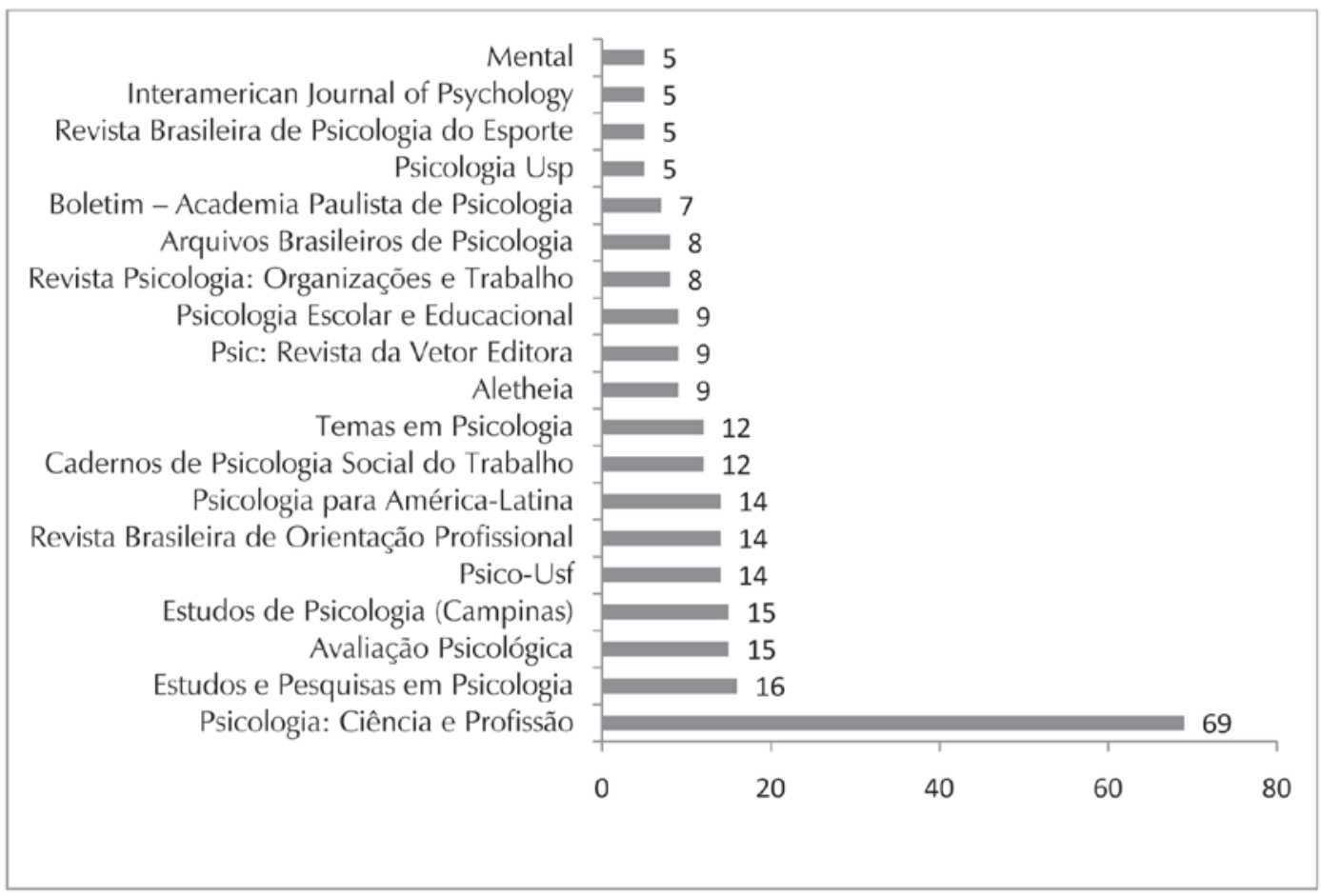

Figura 3. Revistas que mais citaram PCP no período de 2005-2009.

A Figura 3 apresenta um panorama das revistas que citaram PCP no período. A autocitação aparece na revista nos anos estudados. A rede de revistas que mais citam PCP está publicada no PePSIC, demonstrando que publicações eletrônicas ganham maior visibilidade na comunidade científica. Com a entrada de PCP no SciELO, no ano 2010, o panorama deverá ser alterado.

\section{Considerações finais}

O rápido exercício de análise da PCP nos últimos cinco anos permite afirmar que a revista vem alcançando critérios que a qualificam como revista científica de forte relevância para a área. Ao completar 30 anos, a proposta da revista é o comprometimento com a Psicologia voltada para o alívio do sofrimento humano, mas também dedicada ao avanço da ciência psicológica.

O conhecimento documentado em revistas científicas e o impacto que tais publicações representam para o saber universal é algo que deve ser abordado, discutido e entendido. Análises descritivas de publicações científicas, ou do conteúdo nelas publicado, quantitativas ou qualitativas, são meios complementares de compreensão que podem auxiliar a entender o desenvolvimento da ciência em nossos países e devem ser incentivadas como área de estudo (Gutierréz, Pérez-Acosta, \& Plata-Caviedes, 2009). 
Voltamos ao artigo comemorativo dos 25 anos de PCP para, tal como Campos e Bernardes (2005), reiterar os

Parabéns a todos os que participaram desse processo e, em especial, aos psicólogos que, nessas últimas décadas, produziram uma psicologia comprometida com o social, politicamente participante, que escuta todas as camadas sociais e constrói práticas para os diversos campos da vida humana, apoiada no acolhimento das diferenças individuais e socioculturais. (p. 524)

\section{Maria Imaculada Cardoso Sampaio}

Doutorando em Psicologia Experimental, bibliotecária do Instituto de Psicologia da USP/ coordenadora técnica da BVS-psi ULAPSI Brasil e BVS-Psi ULAPSI / professora da Faculdade de Sociologia e

Política de São Paulo, São Paulo - SP - Brasil

E-mail: isampaio@usp.br

\section{André Serradas}

Bacharel em Biblioteconomia e Ciência da Informação pela Faculdade de Biblioteconomia e Ciência da Informação da Fundação Escola de Sociologia e Política de São Paulo Bibliotecário. Membro da Comissão de Credenciamento do Programa de apoio às Publicações Científicas Periódicas da USP, membro fundador da Associação Brasileira de Editores Científicos em Psicologia, Seção de Apoio ao Credenciamento de Revistas USP, Departamento Técnico, Sistema Integrado de Bibliotecas, São Paulo - SP - Brasil

E-mail: serradas@usp.br

\section{Acácia Aparecida Angeli dos Santos}

Doutora em Psicologia Escolar e do Desenvolvimento Humano pela USP

Professora da graduação e da pós-graduação Stricto Sensu em Psicologia da Universidade São Francisco, São

Paulo - SP - Brasil

E-mail: acacia.angeli@gmail.com

\section{Endereço para correspondência:}

Universidade de São Paulo. Instituto de Psicologia. Biblioteca Dante Moreira Leite. Av. prof. Mello Moraes, 1721 - Bl. São Paulo, SP - Brasil CEP 05508-030.

Barreto M. L. (2006). Crescimento e tendência da produção científica em epidemiologia no Brasil. Revista de Saúde Pública, 40(spe), 79-85. Recuperado em 01 de setembro de 2010, de ttp://www.scielo.br/scielo.php?script=sci_ arttext\&pid=S0034-89102006000400012\&lng=en

Campos, R. H. F., \& Bernardes, L. H. G. (2005). A revista Psicologia: Ciência e Profissão: um registro da história recente da Psicologia brasileira. Psicologia: Ciência e Profissão, 25(4). Recuperado em 02 de setembro de 2010, de http://pepsic.bvsalud.org/scielo.php?script $=$ sci arttext\&pid $=$ S1414-98932005000400002\&lng $=$ pt\&nrm $=$ iso

Gutiérrez, G., Pérez-Acosta, A. M., \& Caviedes, T. P. (2009). Desarrollo histórico de una publicación científica: cuarenta años de la Revista Latinoamericana de Psicología. Revista Latinoamericana de Psicología, 41(3), 413-426.

Silva, J. A., \& Bianchi, M. L. P. (2001). Cientometria: a métrica da ciência. Paidéia, 11(21), 5-10. Recuperado em 01 de setembro de 2010, de http://www.scielo.br/scielo. php? script $=$ sci_arttext\&pid $=$ S0103-863X2001000200002\& lng $=$ en \&nrm $=\overline{\text { iso }}$

Volpato, G. L. (2003). Publicação científica (2a ed.). Botucatu, SP: Tipomic. 